ARTículo

\title{
Las playas arenosas de Quintana Roo, México: La diversidad de su fauna intermareal
}

\author{
The sandy beaches of Quintana Roo, Mexico: The intertidal fauna diversity
}

\begin{abstract}
Alicia González ${ }^{1}$, Daniel Torruco ${ }^{1}$ y Ángel D. Torruco²
${ }^{1}$ Centro de Investigación y de Estudios Avanzados del I.P.N., Unidad Mérida, Km 6 Carretera Antigua a Progreso, C.P. 97310, CORDEMEX, Mérida, Yucatán, México

${ }^{2}$ Universidad Anahuac-Mayab, Carretera Mérida-Progreso, km 15.5 C.P. 97310, Mérida, Yucatán, México

Abstract.- Field research of macrofaunal communities of sandy beaches at 10 sites of the central and southern coast of Quintana Roo State, was performed in 2011. For each beach 3 transects were conducted perpendicular to the coast with a variable number of levels depending on the length of the beach. The slopes of the beaches were also variable; however, as a recurring pattern, the southern part of each beach was wider than the northern part. The best represented fauna were mollusks (18 species), but also other groups were recorded such as crustaceans (4), annelids (4), sipunculids (1), nemerteans (1), foraminifera (4) and insects (1). The numerical analysis divided the coast of the state in areas of the Maya Rivera, that were more diverse and richness in fauna, while the sites located in the Gran Costa Maya were the least diverse. The assemblage sites representing the major groups show affinities between nearby sites, but are able to detect differences between areas in the same region. Using a content analysis divisive second-order information, for different groups, the species responsible for most decrease of information was obtained; in mollusks, was Terebra tuberculosa, whereas annelids was Scoloplos latum and for foraminifera Bigenerina angulata. A long-term study is proposed for this ecosystem.
\end{abstract}

Key words: Psammon, spatial distribution, Mayan Riviera, Mayan Great Coast, Mexico

\begin{abstract}
Resumen.- Se realizó una investigación de campo de las comunidades de playas arenosas en 10 sitios al norte y sur del Estado de Quintana Roo, México, en 2011. Para cada playa se realizaron 3 transectos perpendiculares a la costa con un número variable de niveles dependiendo de la longitud de la playa. Las pendientes de las playas fueron también variables; sin embargo, como patrón recurrente, la parte sur de cada playa fue más extensa que la parte norte. La fauna mejor representada fue la de los moluscos (18 especies), aunque también se registraron otros grupos como: crustáceos (4), anélidos (4), sipunculidos (1), nemertinos (1), foraminíferos (4) e insectos (1). Los análisis numéricos dividieron el litoral de la costa en sitios de la Rivera Maya que tuvieron mayor riqueza y diversidad de la fauna y en sitios de ubicados en la Gran Costa Maya que fueron los menos diversos. El ensamblaje que formaron los sitios a partir de los grupos principales muestran afinidades entre sitios cercanos, pero también permitió detectar diferencias entre zonas en una misma región. Utilizando un análisis divisivo del contenido de información de segundo orden, para los diferentes grupos, se obtuvo las especies responsables de la mayor caída de información; en los moluscos, fue Terebra tuberculosa, mientras que para los anélidos fue Scoloplos latum y para los foraminiferos Bigenerina angulata. Se propone un estudio a mayor plazo para este ecosistema.
\end{abstract}

Palabras clave: Psammofauna, distribución espacial, Riviera Maya, Gran Costa Maya, México

\section{INTRODUCCIÓN}

Por las características intrínsecas de su naturaleza, el turismo es la mayor actividad del litoral de Quintana Roo, México. Los productos y servicios están supeditados al principal activo ambiental que son las caletas y los sistemas playa-duna, de los que depende parte de la economía del estado. Actualmente, la gestión de playas en esta zona se ha limitado a dos vías: los programas de recuperación (Batllori 2012) y la preocupación de cumplir con las normas básicas de higiene e instalación de ciertos servicios destinados al ocio y la recreación. Esta segunda con la finalidad de la obtención de eco-etiquetado y certificaciones de playa limpia, segura y otros procesos de gestión recientemente adoptados para estos sistemas litorales. El Estado de Quintana Roo, ha obtenido su certificación en 25 de las 27 solicitudes sometidas hasta 2011 (Fideicomiso de Promoción Turística Rivera Maya 2011¹, CONAGUA 2007$2012^{2}$ ).

${ }^{1}$ Fideicomiso de Promoción Turística Riviera Maya. 2011. Certificación AENOR ISO 14001 para las playas de la Riviera Maya. Reseña rueda de prensa.

${ }^{2}$ CONAGUA. 2007-2012. Guía esquemas para la conservación del suelo, bosque y agua. Serie: Planeación hidráulica en México. SEMARNAT, $92 \mathrm{pp}$ 
Las playas arenosas representan uno de los ambientes marinos más severos para las comunidades de animales macrobentónicos, ya que éstas se encuentran controladas por procesos físicos (oleaje y corrientes) y geológicos (origen, sedimentación, tamaño de grano y transporte de sedimentos) que moldean la costa (Torruco \& González-Solís 2012). Estos espacios litorales pertenecen a sistemas frágiles y dinámicos sobre los que intervienen un gran número de factores de diferente naturaleza y magnitud. Las playas son entendidas comoun anexo más a los espacios turístico-hoteleros y urbanos, obviando sus características ambientales, las funciones de reservas sedimentológicas, las morfologías de sus dunas asociadas y que constituyen el hábitat de numerosas especies adaptadas a estos ambientes (Schmitt 1994). La distribución de la psammofauna en las playas arenosas es heterogénea con parches y fluctuaciones que son debidas a la marea y a las migraciones de los organismos (McLachlan \& Hesp 1984), el tipo de sustrato, el período de permanencia sumergida y el viento, son factores que controlan en mayor o menor medida esa distribución (Burcharth et al. 2007). Bajo este contexto, el objetivo de este trabajo fue caracterizar la fauna intermareal de 10 playas del Estado de Quintana Roo, su distribución y dependencia de la pendiente de la playa, lo que proporciona un marco de referencia ante los cambios que podría originar la demanda masiva y los posibles perjuicios para la conservación y estabilidad de los ecosistemas litorales (Martín \& RodríguezPerea 1996, Roig 2003, Pérez-Ruíz 2012, Ramírez-Torres 2012).

\section{MATERIALES Y MÉTODOS}

\section{Área de ESTUdio}

El Estado de Quintana Roo tiene una línea de costa cuya formación geológica es de naturaleza caliza que está erosionada extensivamente y proporciona numerosas playas arenosas y espolones rocosos. Los sitios de muestreo se localizan tanto en la zona de la Riviera Maya como en la parte sur del Estado, la Gran Costa Maya, desde Puerto Morelos hasta Xcalak (19 $1^{\circ}$ ' a $20^{\circ} 50^{\prime} \mathrm{N} ; 86^{\circ} 53^{\prime}$ a $87^{\circ} 35^{\prime} \mathrm{W}$ ) (Fig. 1). El área forma parte de la segunda barrera arrecifal más grande del mundo. Esta estructura representa una protección natural contra el oleaje, favoreciendo la presencia de playas poco inclinadas y olas de baja energía (Torruco et al. 2013). Estos factores intervienen dirigiendo los cambios rápidos de circulación y establecen el flujo de sedimentos.

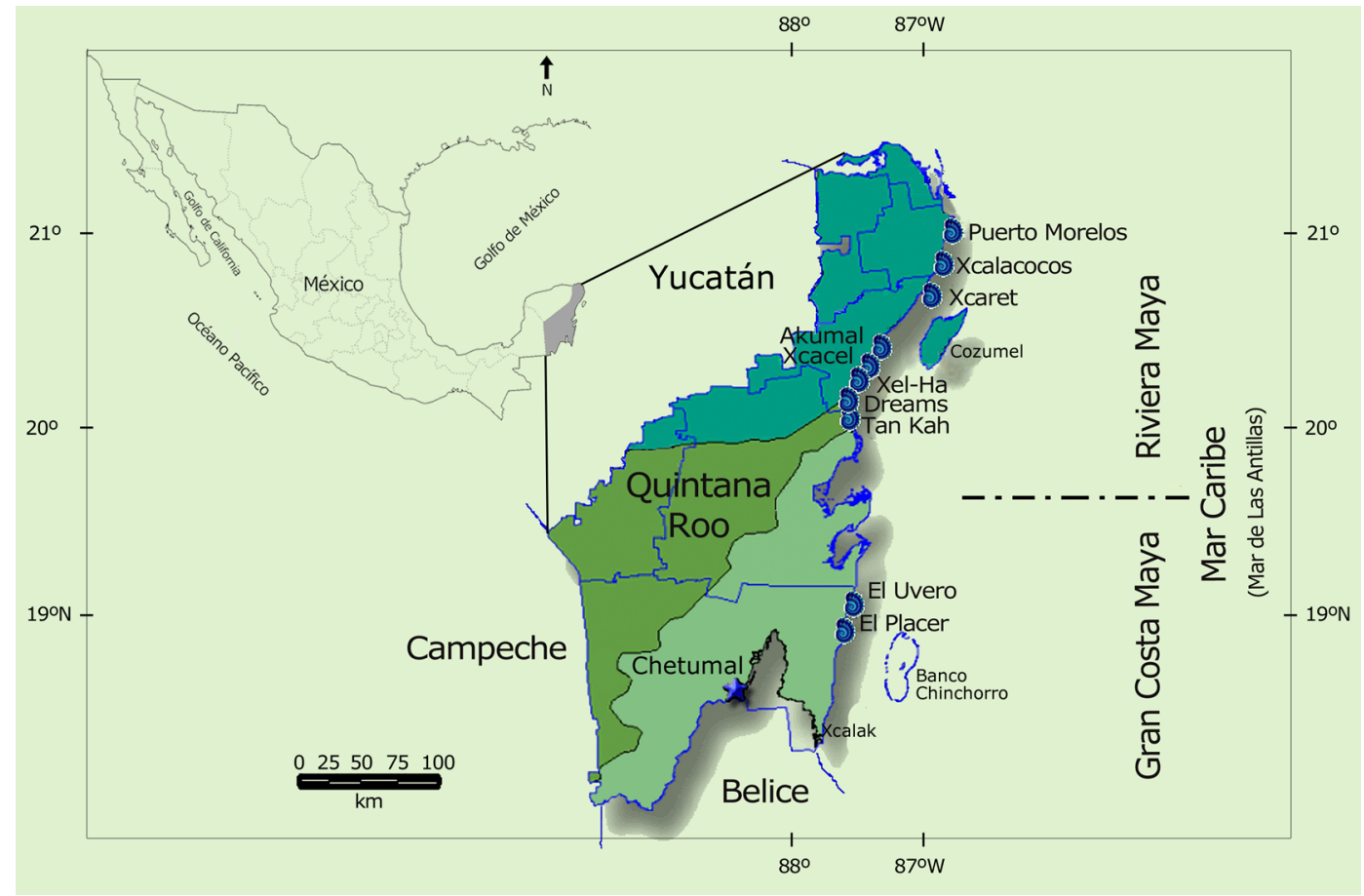

Figura 1. Ubicación de los sitios de estudio en el Estado de Quintana Roo, México / Location of the study sites in the Quintana Roo State, Mexico 
En cada localidad, se realizaron 3 transectos perpendiculares al mar. La distancia entre las transectas fue relativa al tamaño de la playa, de manera tal que se realizó uno al norte, uno al centro y uno al sur de cada playa. El número de muestras estuvo definido por los límites impuestos por la humedad y por la fuerza del oleaje, siendo los más cortos de 6 niveles y los más largos de 11 .

\section{Estudio físico}

La temperatura de los sitios muestreados se tomó a $5 \mathrm{~cm}$ de profundidad y en la superficie del sedimento, con un termómetro de mercurio, con precisión de $1^{\circ} \mathrm{C}$. Con las réplicas de cada muestra se tomaron alícuotas de $200 \mathrm{~g}$, para determinar la granulometría por el método de Bouyoucos (Cano 2010) y la materia orgánica por pérdida de peso de las muestras de sedimento por ignición en una mufla a $450^{\circ} \mathrm{C}$. La pendiente de la playa en los transectos se obtuvo con un nivel topográfico y los puntos de muestreo fueron geoposicionados con un GPS Garmin 12LX.

\section{Estudio biológico}

Inicialmente, se realizó una aproximación que implica tanto el número de muestra como el área mínima que cubrirán las muestras para que sean estadísticamente representativas de las localidades mencionadas. En un muestreo prospectivo, tomando 2 playas al norte y dos al sur, se analizaron diferentes volúmenes de muestras para determinar el número de especies presentes en cada una de ellas y con ello se estableció una curva de número de especies-volumen de arena, seleccionando un volumen de $251,328 \mathrm{~cm}^{3}$, valor que alcanzó la curva al volverse una asíntota. Las muestras fueron recolectadas a lo largo de cada transecto, la distancia entre cada punto fue de 3 m. Para el inicio del transecto se utilizó la línea de humedad que corresponde al inicio de la vegetación pionera de la duna y el final fue determinado por el oleaje. Las muestras fueron tomadas en las mañanas (6:00-9:00 AM) cuando aún la insolación no es total. Para cada nivel se tomaron tres réplicas con un nucleador de $20 \mathrm{~cm}$ de largo y un diámetro de $14 \mathrm{~cm}$, que fueron colocadas en bolsas de plástico herméticas. La muestra biológica fue fijada con FAA (Formol-Alcohol-Ac. Acético) y Rosa de Bengala para colorear los organismos vivos. Posteriormente, se trasladó al laboratorio para su lavado, tamizado automático por un tamiz de $1 \mathrm{~mm}$ y preservación en alcohol al $70 \%$. Cada grupo fue identificado hasta el nivel taxonómico más bajo posible utilizando bibliografía especializada (Warmke \& Abbot 1962, Gibson 1972, Cutler 1973, Felder 1973, Fauchald 1977, Powers 1977, Stephen \& Edmonds 1977, Williams 1984, Loeblich \& Tappan 1988, Abbot 1996), conformando después una matriz de abundancia que fue sometida a los análisis estadísticos siguientes:

\section{ANÁLISIS NUMÉrico}

El análisis numérico incluyó la obtención de parámetros comunitarios como dominancia (Índice del valor de Importancia) y diversidad (Índice de Shannon-Wiener) (Magurran 1988) y el uso de estadística multifactorial en técnicas de clasificación jerárquica con el coeficiente de BrayCurtis, con un tipo de unión flexible con un $\beta=-0,2$, en 3 de los grupos con mayor abundancia, para determinar el ensamblaje de los sitios (Orlocí 1978, Pielou 1984). Para definir las especies que pudieran ser utilizadas como indicadoras de cambios, la matriz binaria de presencia y ausencia, se sometió a un análisis exploratorio del contenido de información de segundo orden (modificado de Ezcurra \& Equihua 1984) bajo un procedimiento monotético divisivo para identificar las especies que discriminan cada una de las caídas de información del conjunto de datos y obtener con ello las especies indicadoras de cada localidad, la fórmula del estadístico es la siguiente:

$$
C I_{2}=2 S n \log n-n \sum_{i=1}^{s} \log \left[a_{i}^{2}+\left(n+a_{i}\right]^{2}\right.
$$

donde:

$S=$ Número de descriptores $\left(\mathrm{N}^{\circ}\right.$ Especies $)$

$n=$ Número de objetos ( $\mathrm{N}^{\mathrm{o}}$ de sitios)

$a_{i}=$ Número de objetos donde está presente el descriptor $i$

El procedimiento utilizado para la obtención de especies indicadoras tiene como base la diversidad de especies de cada sitio para cada grupo. Se realizó la clasificación monotética directamente sobre los datos originales e inicialmente calcula el contenido de información de orden 2, para todas las combinaciones posibles por un método iterativo. Así, se evalúa el cambio en este estadístico debido al agrupamiento de los sitios de manera, que aquellos lugares con todas las combinaciones posibles para los que el cambio es menor al unirse formarán un grupo. El descriptor que produce el máximo cambio al dividir un conjunto en dos grupos con la mayor homogeneidad posible, se elige como discriminante; la expresión de decisión es la siguiente:

donde:

$$
\text { Máx } \Delta l_{i}=I_{t}-I_{p}-I_{a}
$$

$i=$ descriptor discriminante,

$I_{t}=$ información en el conjunto original,

$I_{p}=$ información para el grupo donde $i$ está presente y

$I_{a}^{p}=$ información para el grupo donde $i$ está ausente.

El proceso continúa hasta que los grupos no pueden ser subdivididos, que coincide en este caso con los sitios. 

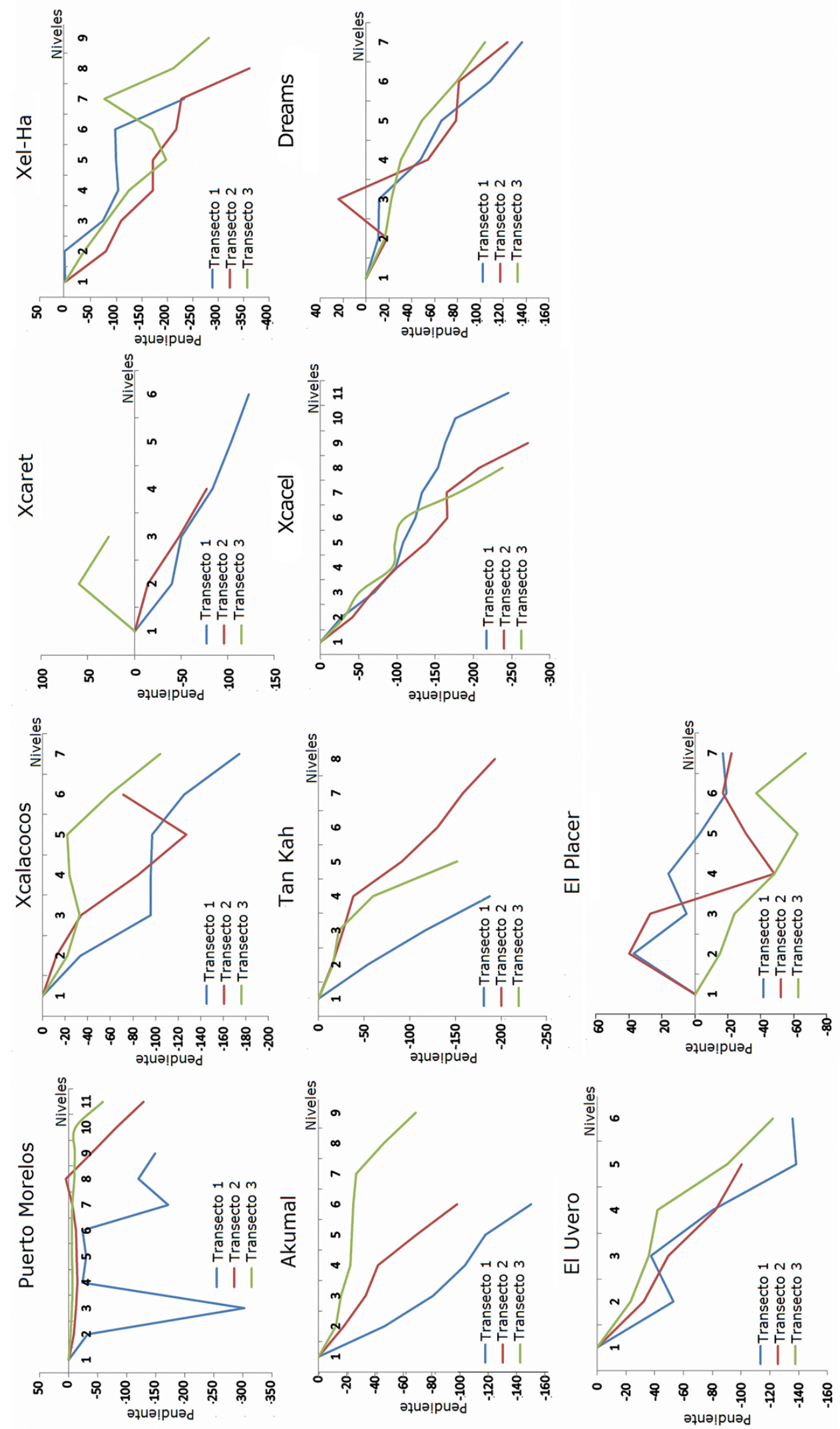
Tabla 1. Promedios granulométricos de las playas arenosas de Quintana Roo / Grain size average of the Quintana Roo sandy beaches

\begin{tabular}{|c|c|c|c|c|c|c|}
\hline Localidad & $\mathrm{LN}$ & LW & Clasificación & $\begin{array}{c}\text { Esfericidad } \\
(\%)\end{array}$ & $\varnothing$ & Curtosis \\
\hline Puerto Morelos & $20^{\circ} 50^{\prime} 15^{\prime \prime}$ & $86^{\circ} 53^{\prime} 4^{\prime \prime}$ & Arenas medias & 62,5 & $0,75-1,02$ & Platicurtico \\
\hline Xcalacoco & $20^{\circ} 39^{\prime} 42^{\prime \prime}$ & $87^{\circ} 1^{\prime} 56^{\prime \prime}$ & Arenas medias-finas & 66,4 & $1,75-2,30$ & Mesocurtico \\
\hline Xcaret & $20^{\circ} 34^{\prime} 42^{\prime \prime}$ & 87'9', & Arenas gruesas-medias & 56.2 & $0,77-1,50$ & Platicurtico \\
\hline Akumal & $20^{\circ} 23^{\prime} 28^{\prime \prime}$ & $87^{\circ} 18^{\prime} 56^{\prime \prime}$ & Arenas medias-finas & 72,0 & $1,50-2,00$ & Platicurtico \\
\hline Xcacel & $20^{\circ} 20^{\prime} 19^{\prime \prime}$ & $87^{\circ} 20^{\prime} 50^{\prime \prime}$ & Arenas-medias & 67,2 & $1,30-2,00$ & Mesocurtico \\
\hline Xel-Ha & $20^{\circ} 18^{\prime} 49^{\prime \prime}$ & $87^{\circ} 21^{\prime} 18^{\prime \prime}$ & Arenas medias & 64,6 & $1,50-2,10$ & Mesocurtico \\
\hline Dreams & $20^{\circ} 13^{\prime} 54^{\prime \prime}$ & $87^{\circ} 25^{\prime} 4^{\prime \prime}$ & Arenas medias-finas & 68,4 & $1,70-2,20$ & Mesocurtico \\
\hline Tan Kah & $20^{\circ} 15^{\prime} 46^{\prime \prime}$ & $87^{\circ} 23^{\prime} 37^{\prime \prime}$ & Arenas medias-finas & 67,9 & $1,70-2,60$ & Platicurtico \\
\hline El Placer & $18^{\circ} 53^{\prime} 21^{\prime \prime}$ & $87^{\circ} 38^{\prime} 27^{\prime \prime}$ & Arenas gruesas-medias & 55,7 & $0,82-1,80$ & Leptocurtico \\
\hline El Uvero & $19^{\circ} 1 ’ 30^{\prime}$, & $87^{\circ} 35^{\prime} 1{ }^{\prime}$, & Arenas gruesas-medias & 58,8 & $0,81-1,75$ & Leptocurtico \\
\hline
\end{tabular}

\section{Resultados}

Estudio físico. Las pendientes de las playas analizadas fueron semejantes, con excepción de las playas de Puerto Morelos, Xcacel y Xel-Ha, donde descendieron más de los 2,5 m; en algunos sitios, la distancia horizontal tuvo diferencias de hasta 5 niveles. Todas ellas presentaron pendientes suaves poco abruptas; no hubo diferencias notables en cuanto a la longitud de los transectos posicionados hacia el norte o hacia el sur de cada playa, el transecto central fue de longitud intermedia entre la parte sur y la parte norte (Fig. 2).

La temperatura del substrato no tuvo variaciones notorias en ninguna de las playas, tanto en superficie como a $5 \mathrm{~cm}$ de profundidad, aun cuando a esta profundidad los valores fueron ligeramente más altos $\left( \pm 1,0^{\circ} \mathrm{C}\right)$. Aunque el gradiente de humedad en el sedimento fue en incremento desde el nivel superior (inicio de los transectos), hasta el nivel inferior de la zona intermareal, no hubo una separación clara en zonas que fueran definidas por la cantidad de agua en cada playa. El contenido de materia orgánica en cada playa, no mostró un patrón de distribución por marea y fueron menores de $0,3 \%$ y en ninguna de las muestras se observaron sedimentos reducidos (capa obscura). En cuanto a la granulometría, todos los sitios tuvieron un $100 \%$ de arena (sin limos ni arcillas), generalmente de arenas medias a finas (Tabla 1). En algunas playas se observó un mayor porcentaje de residuos de organismos. No se diferenciaron significativamente en términos de grano medio, esferocidad y curtosis (prueba de $\mathrm{t}$ pareada, $\mathrm{t}=3,057, P=$ 0,028).

Estudiobiológico. La macrofauna en las playas de Quintana Roo estuvo integrada por 7 grupos: moluscos, crustáceos, anélidos, sipuncúlidos, nemertinos, foraminíferos e insectos (Tabla 2). De estos, el grupo de los moluscos (16 gasterópodos y 2 bivalvos) fue el de mayor número de especies $(54,54 \%)$.
Tabla 2. Riqueza de especies y abundancia relativa de la psammofauna en las playas de Quintana Roo, México / Richness and relative abundance of the psammofauna on the beaches of Quintana Roo, Mexico

\begin{tabular}{lcc}
\hline \multicolumn{1}{c}{ Grupos } & $\begin{array}{c}\text { Riqueza de } \\
\text { especies }\end{array}$ & $\begin{array}{c}\text { Porcentaje } \\
(\%)\end{array}$ \\
\hline Mollusca & 18 & 54,54 \\
Crustacea & 4 & 12,12 \\
Annelida & 4 & 12,12 \\
Foraminifera & 4 & 12,12 \\
Nemertea & 1 & 3,03 \\
Sipuncula & 1 & 3,03 \\
Insecta & 1 & 3,03 \\
\hline
\end{tabular}

En cuanto a la abundancia, los foraminíferos dominaron, constituyendo un total de $59,60 \%$, seguido de los moluscos $(17,99 \%)$ y los demás grupos con una abundancia relativa muy baja. A pesar de la dominancia, moluscos y foraminíferos estuvieron ausentes en las playas de la Gran Costa Maya, donde se registró mayor tamaño de grano y residuos de esqueletos de organismos. Si se considera la riqueza de especies por transecto por playa, la mayor riqueza se presentó en los transectos más largos, siendo esto un reflejo del aumento en el número de muestras (prueba Chi Cuadrado, $P<0,01$ ). Se encontró un total de 33 especies en las 10 playas (Anexo 1).

En una vista diagramática de la distribución de la densidad de los moluscos, el arreglo espacial del grupo fue en la parte media de los transectos, con excepción de Xcaret, que es una playa artificial y su ausencia en la Gran Costa Maya es sorprendentemente notable (Fig. 3). Los crustáceos estuvieron presentes en 3 localidades, dos de ellas en la Gran Costa Maya donde se encontraron sus mayores abundancias en la parte media de la playa. Los anélidos tuvieron un patrón semejante a 

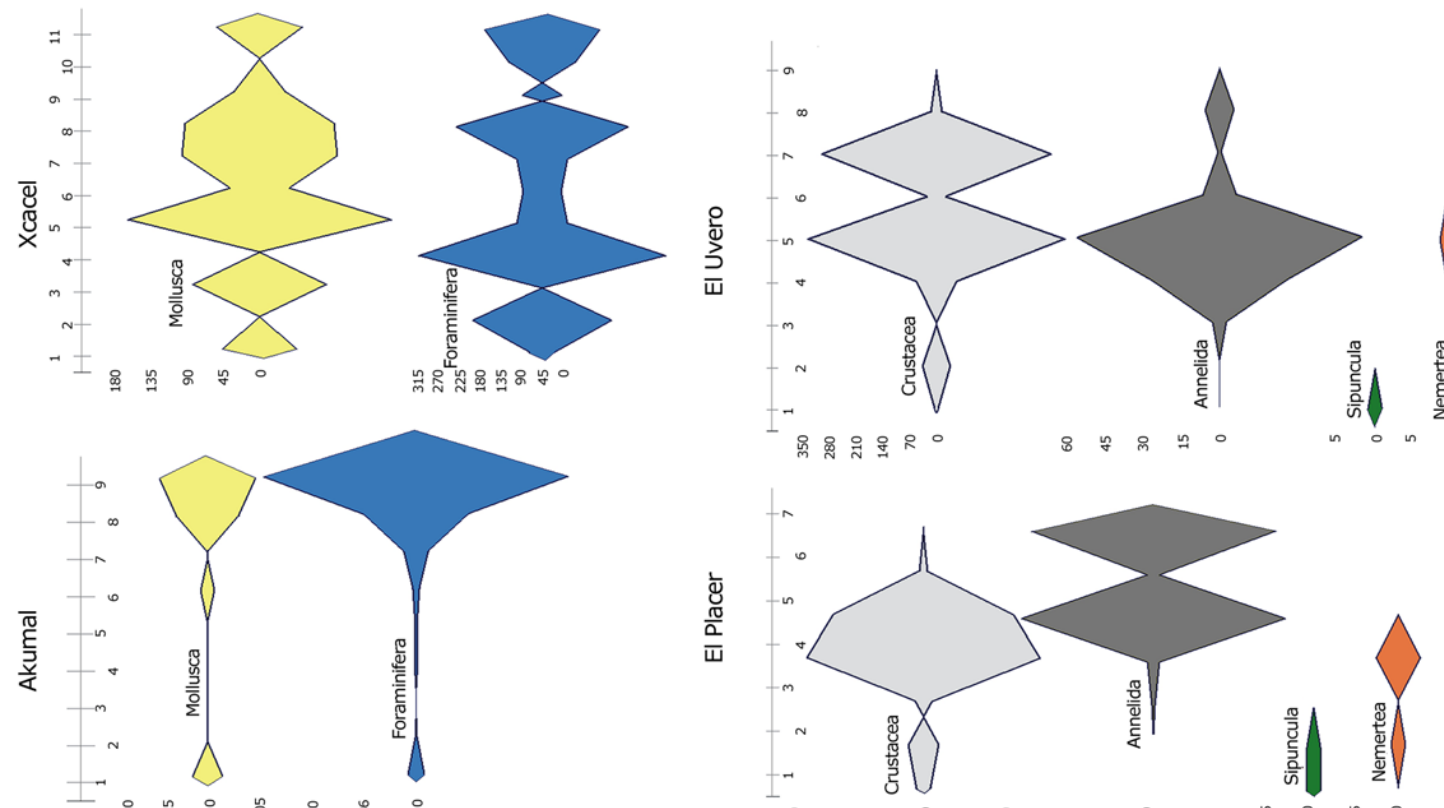

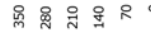
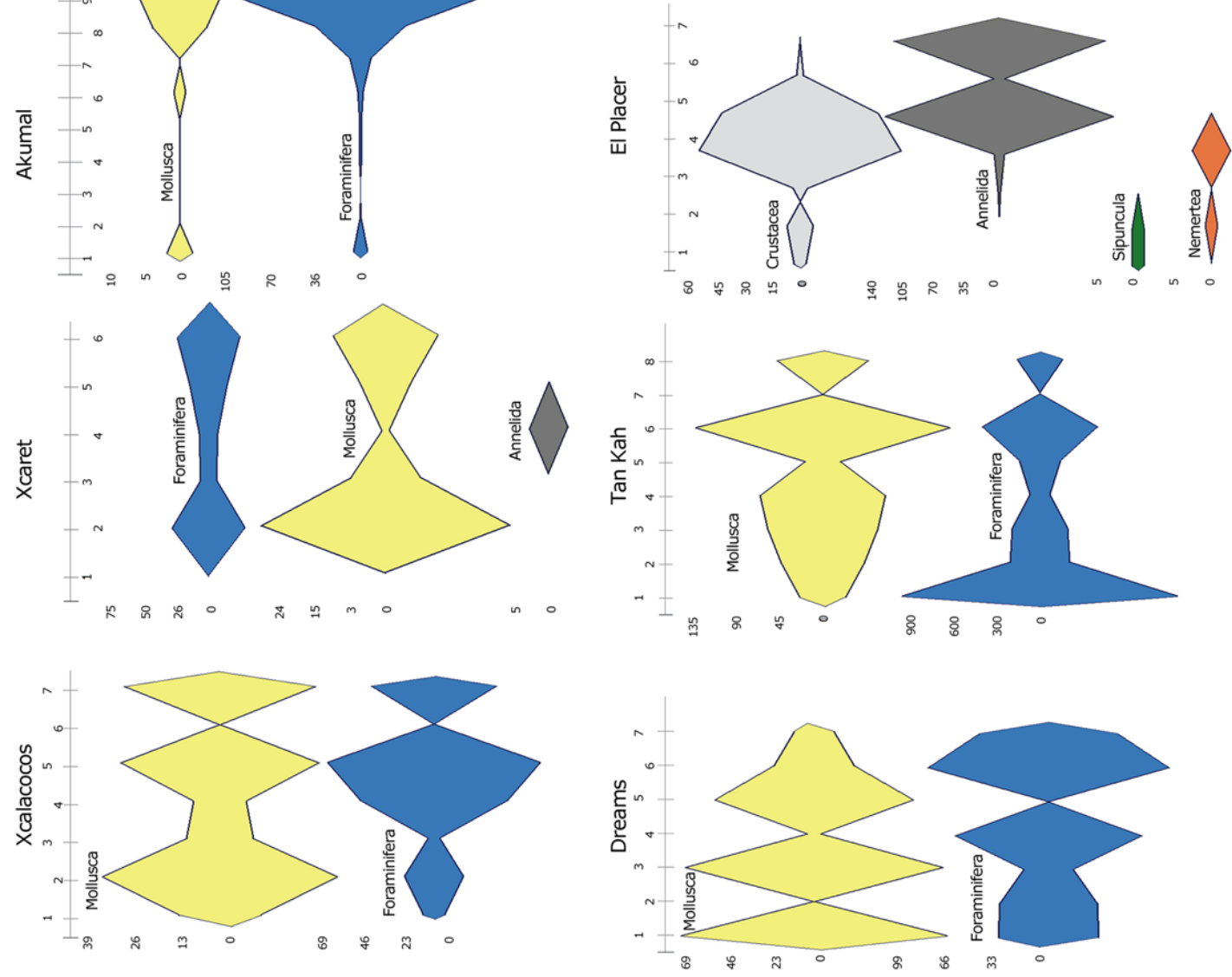

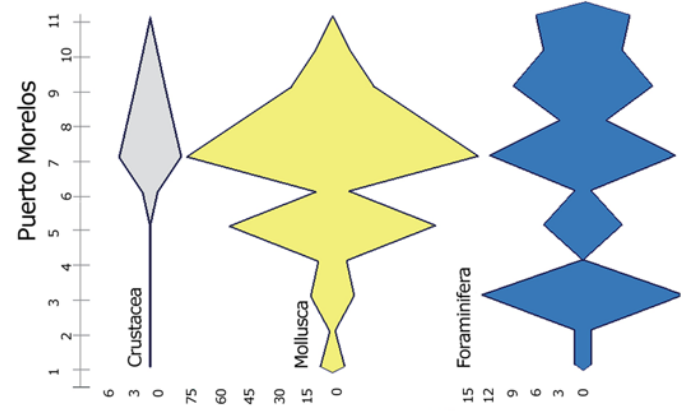

eIouepunq

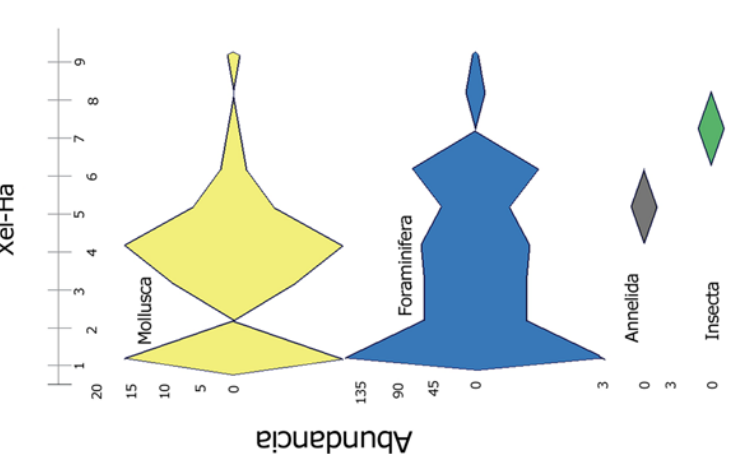

uepunq $\forall$ 
los crustáceos, con mayores densidades en las estaciones El Placer y El Uvero en la Gran Costa Maya. Los sipuncúlidos estuvieron presentes en dos estaciones de la Gran Costa Maya, al inicio de los transectos. Los nemertinos se distribuyeron en la Gran Costa Maya con bajas densidades, desde el inicio del transecto hasta la parte media de la playa. Los foraminíferos fue un grupo dominante a lo largo de los transectos, pero estuvieron ausentes en El Placer y El Uvero de la Gran Costa Maya (Fig. 3).

El índice de dominancia (IVI), no tuvo una diferencia marcada entre las 2 especies predominantes de cada grupo, pero que puede ser muy marcada entre la primera y tercera. Entre ellas se reúne más del 50\% de la dominancia total del grupo correspondiente (Tabla 3 ).

Con relación a la riqueza de especies, en los sitios de la Rivera Maya, los moluscos fue el grupo con mayor número, seguido de los foraminíferos. Los crustáceos tuvieron la mayor riqueza en los sitios de la Gran Costa Maya (Fig. 4a).

La diversidad fue más alta para los moluscos, alcanzando su máximo en Akumal (2,75 bits ind $\left.{ }^{-1}\right)$ y la menor fue en Puerto Morelos $\left(0,93\right.$ bits ind $\left.^{-1}\right)$. Los foraminíferos tuvieron su mayor diversidad en Xcalacoco (1,35 bits ind $\left.{ }^{-1}\right)$, mientras que la más baja se observó en Puerto Morelos (0,32 bits ind $^{-1}$.). Con los crustáceos la mayor diversidad se registró en El Placer $\left(1,85\right.$ bits ind $\left.^{-1}\right)$ y la menor en Puerto Morelos $\left(0,56\right.$ bits ind $\left.^{-1}\right)$ (Fig. 4b).

El análisis de clasificación numérica de los sitios con respecto a cada grupo evidenció las afinidades que se muestran en un corte al 50\%. Para los moluscos a partir de registros en 8 sitios de la Riviera Maya, mostró 2 grupos y una localidad solitaria (Akumal). Uno de los grupos fue conformado por las playas Xcaret y Xel-Ha, que son parques de alta afluencia turística. El otro grupo incluyó las playas restantes, a niveles más bajos

Tabla 3. Especies con mayor Índice de Valor de Importancia (IVI) en los diferentes grupos de las playas arenosas del litoral de Quintana Roo / Species with higher Importance Value Index (IVI) in the different groups of sandy beaches along the coast of Quintana Roo

\begin{tabular}{lc}
\hline \multicolumn{1}{c}{ Grupos } & IVI $(\%)$ \\
\hline Crustacea & \\
Talitrus saltator & 35,55 \\
Eurydice convexa & 24,46 \\
$\quad$ Gammarus annulatus & 20,34 \\
Mollusca & \\
Rissoella caribaea & 23,37 \\
Parvanachis obesa & 20,4 \\
Bentonella tenella & 18,5 \\
Foraminifera & \\
Archaias agulatus & 45,05 \\
Cyclorbiculina compressa & 31,62 \\
Homotrema rubra & 18,09 \\
\hline
\end{tabular}

este grupo reveló fuerte asociación entre playas del norte y playas del sur de la Riviera Maya (Fig. 5a). Los crustáceos mostraron sólo alta afinidad con sitios del sur y una localidad solitaria en la Riviera Maya norte (Fig. 5b). Los anélidos manifestaron fuertes similitudes entre sitios de la Riviera Maya y entre sitios de la Gran Costa Maya (Fig. 5c). Los foraminíferos mostraron fuertes afinidades en localidades cercanas como es el caso de Xcacel y Dreams, pero también une sitios más distantes (Fig. 5d).

Las especies indicadoras del grupo de los moluscos están representadas tanto por la clase gasterópoda como bivalvia; no obstante que el gasterópodo Terebra tuberculosa fue el
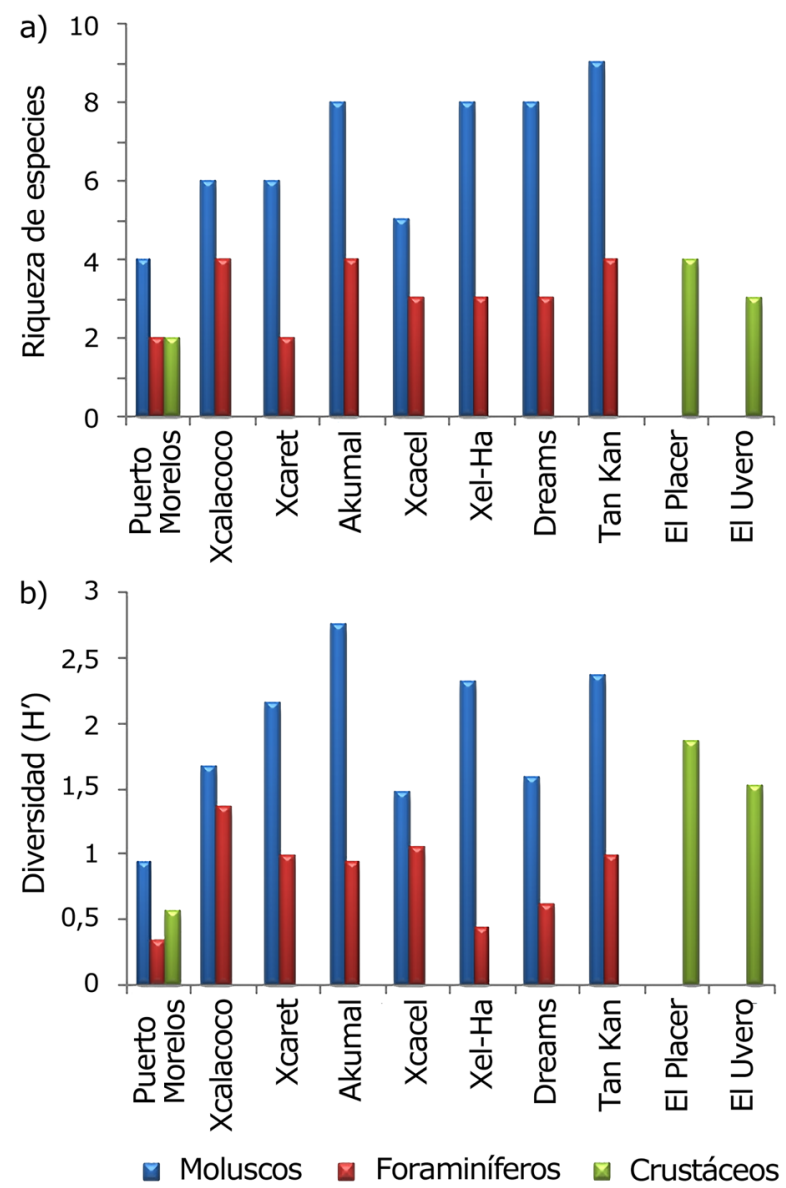

Figura 4. Diversidad de grupos faunísticos en las playas arenosas: a) Riqueza de especies, los moluscos es el grupo con mayor número de especiesen todos los sitios de la Riviera Maya, b) Diversidad ecológica, los moluscos tienen la mayor diversidad en casi todos los sitios, con excepción de El Uvero y El Placer, sitios de la Gran Costa Maya y donde son desplazados por las Crustáceos / Diversity of sandy beach faunal groups: a) Species richness, mollusks is the group with the highest species number in all sites of the Mayan Riviera, b) Ecological diversity, mollusks have the most diversity in most sites, except in the El Uvero and El Placer where they are displaced by crustaceans 
causante de la mayor caída de información. Los crustáceos Gammarus sp. discriminaron los sitios de la Gran Costa Maya de cada sitio; Telitrus saltator fue el responsable de la mayor caída de información. En los anélidos Arenicola sp. discriminó a las localidades de la Riviera Maya y Scoloplos latum (Chamberlin, 1919) a las de la Gran Costa Maya, siendo este último el responsable de la mayor caída de información. Los foraminíferos Homotrema rubrum y Archaias compresus, discriminaron a las 8 localidades de la Riviera Maya (Tabla 4).

La presencia y ausencia de las especies de moluscos revelaron una división entre los sitios norte y sur de la Riviera Maya, presentando asociaciones estrechas en áreas colindantes
(Fig. 6a). Los crustáceos separaron los sitios de la Riviera Maya con los de la Gran Costa Maya y unen fuertemente los sitios de estos últimos (Fig. 6b). Los anélidos presentaron un patrón semejante, separando determinantemente los sitios de la Rivera Maya y de la Costa Maya y uniendo los sitios afines (Fig. 6c). Los foraminíferos fueron más frecuentes, pero aun cuando su distribución se restringió solo a la Riviera Maya, manifestaron uniones fuertes entre sitios cercanos, lo cual muestra la afinidad en la presencia de su fauna, la especie Bigenerina angulata Cushman, 1927 fue la responsable de la mayor caída de información en este grupo (Fig. 6d).

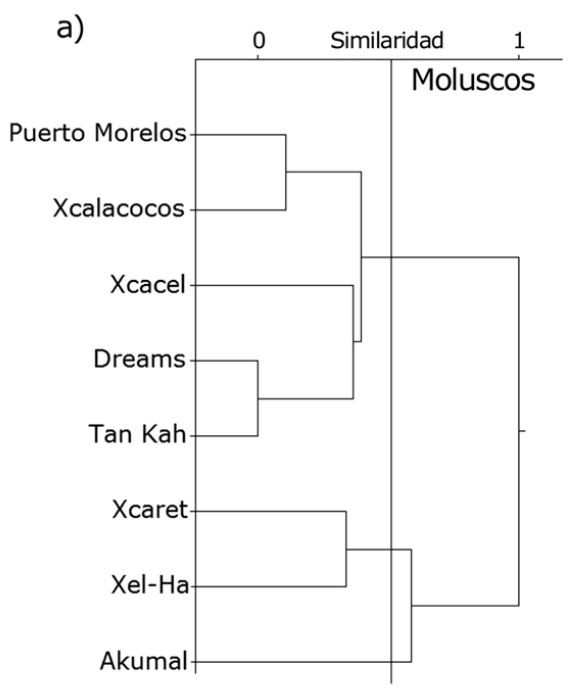

b)

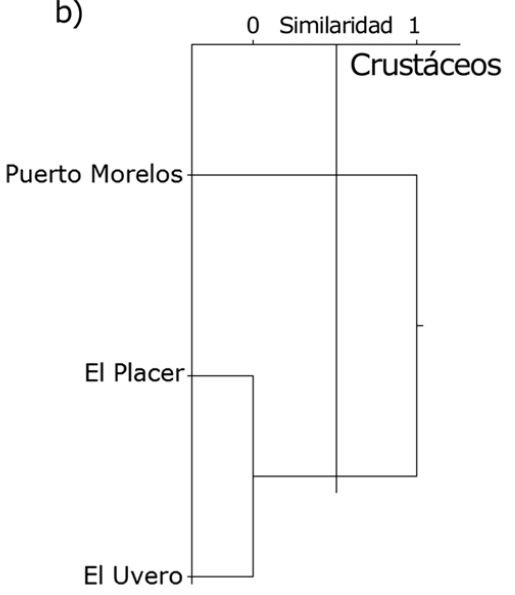

c)

0 Similaridad 1
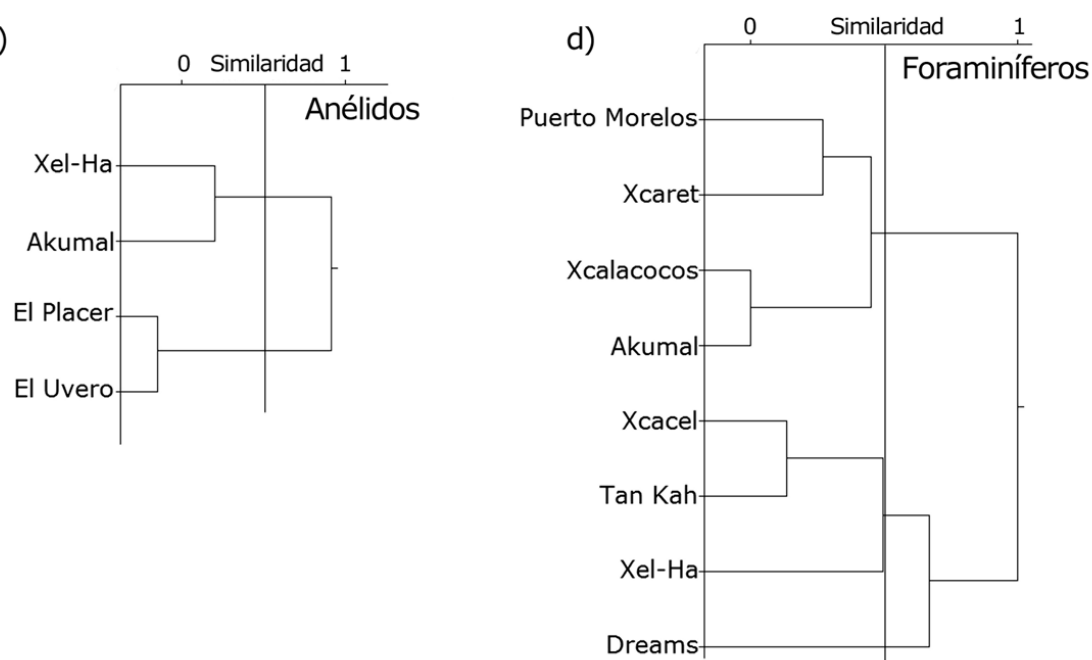

Figura 5. Dendrogramas de los sitios basados en la similitud de la abundancia de los diferentes grupos. a) Moluscos, b) Crustáceos, c) Anélidos y d) Foraminíferos. Se muestran las asociaciones en sitios cercanos y la separación entre sitios de la Riviera Maya y de la Gran Costa Maya / Clusters showing the sites association based on the abundance similarity of the different groups. a) Mollusks, b) Crustaceans, c) Annelids and d) Foraminifera. Strong association between nearby sites and the spacing between sites of the Mayan Riviera Maya and the Great Mayan Coast are present 
Tabla 4. Especies discriminantes, contenido de información de orden $2\left(\mathrm{Cl}_{2}\right)$ y caída de información que representan para cada grupo / Discriminating species, order 2 information content $\left(\mathrm{Cl}_{2}\right)$ and information fall for each group

\begin{tabular}{llcc}
\hline \multicolumn{1}{c}{ Grupo } & Especie discriminante & $\mathrm{CI}_{2}$ Total & Caida de información \\
\hline Mollusca & Terebra tuberculosa & 61,79 & 17,84 \\
Crustacea & Talitrus saltator & 5,29 & 3,9 \\
Annelida & Scoloplos latum & 4,35 & 2,93 \\
Foraminifera & Homotrema rubra & 11,28 & 5,64 \\
\hline
\end{tabular}

a)

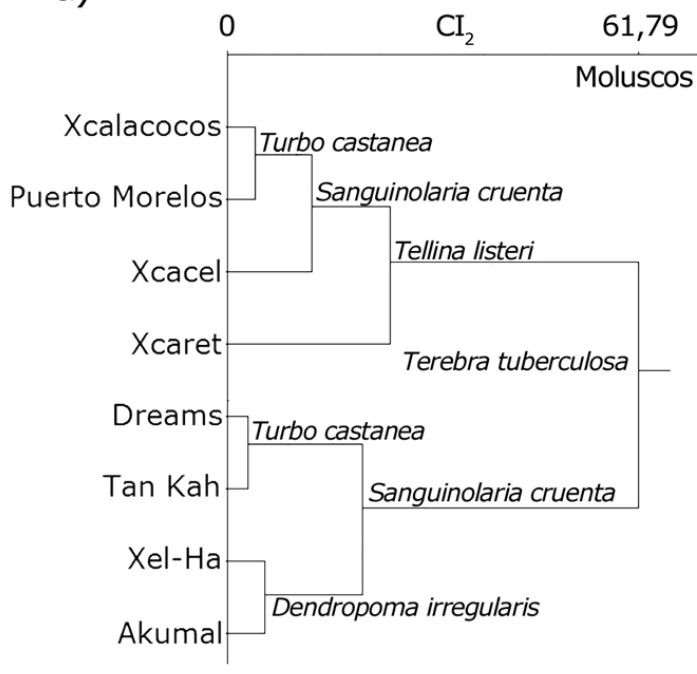

c)

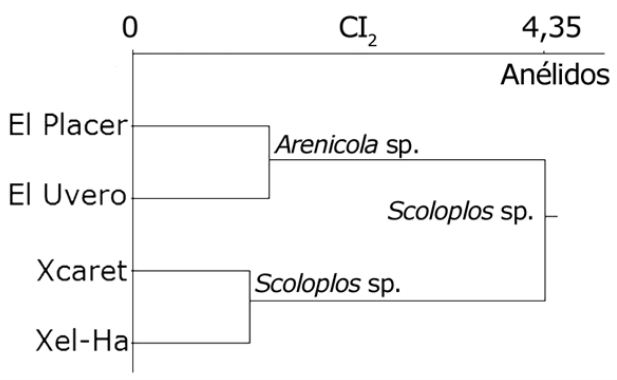

\section{Discusión}

En las últimas décadas, la necesidad de proteger asentamientos humanos y negocios productivos amenazados por la erosión costera, así como el incremento en la utilización de las playas con fines recreativos, han llevado a un mayor interés por el conocimiento de los procesos que actúan en la dinámica litoral de ese ecosistema.

El análisis de los sedimentos obtenidos en las playas arenosas de las localidades estudiadas, da evidencia de que dichos sedimentos se encuentran formados por restos de corales, moluscos, algas, foraminíferos, briozoos, equinodermos, crustáceos, poríferos y anélidos Los foraminíferos encontrados b)

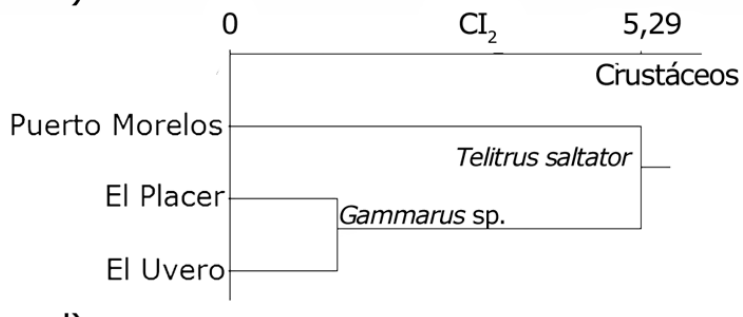

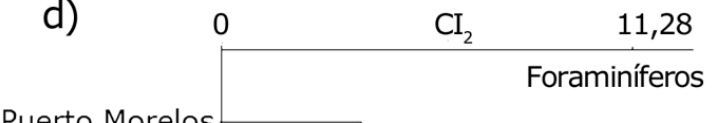

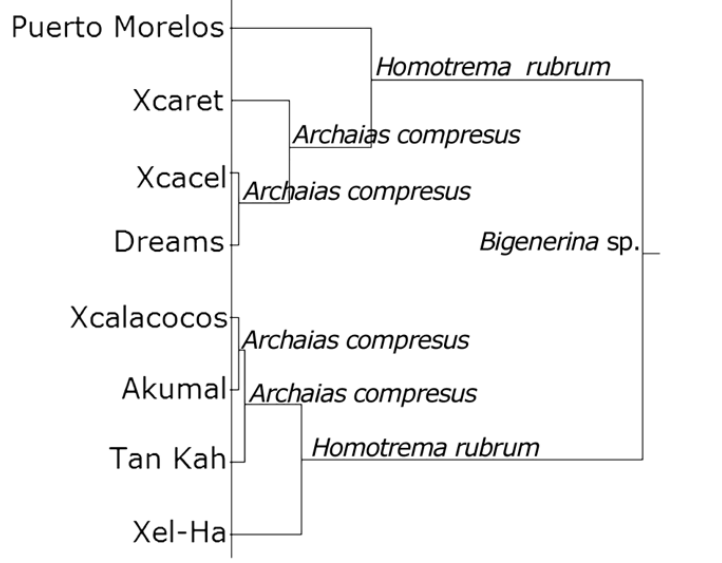

Figura 6. Dendrogramas del $\mathrm{Cl}_{2}$ total para cada grupo y la especie responsable de la principal caída de información. a) M oluscos, b) Crustáceos, c) Anélidosy d) Foraminíferos. Algunos grupos (anélidos, crustáceos) confirman la separación de los sitios entre la Riviera Maya y la Gran Costa Maya / Clusters showing $\mathrm{Cl}_{2}$ total for each group and the main species responsible for information falling. a) Mollusks, b) Crustaceans, c) Annelids and d) Foraminiferal. Some groups (Annelida, Crustacea) confirmed the separation of the sites from the Mayan Riviera and the Great Mayan Coast 
fueron casi en su totalidad aporcelanados (formas que requieren de alta disponibilidad de carbonato de calcio y alta temperatura) y algunos hialinos (formas con requerimientos intermedios) según De Huelbes et al. (1998). No se encontraron aglutinados (formas que se desarrollan en bajas salinidades y baja temperatura con poca disponibilidad de carbonato de calcio). Así, la distribución de los foraminíferos bentónicos en estos tres grupos está determinada por la disponibilidad de carbonato de calcio disuelto en el agua marina (Greiner 1970) ya que estos organismos construyen sus testas de este material.

Las características granulométricas probablemente indiquen una etapa de transición de las condiciones sedimentarias caracterizadas por la erosión y reposición de los substratos antiguos que afloran en el margen litoral y por la gran cantidad de fragmentos de diferentes texturas de origen biogénico. Este último puede ser el responsable del aumento o disminución del coeficiente de selección y del diámetro medio de las partículas; aunado con el poco aporte terrígeno por parte de ríos y escorrientías o de grandes desarrollos costeros (CarranzaEdwards \& Nolasco-Montero 1988).

Aun cuando el grupo de los moluscos, tuvo una amplia distribución a lo largo del perfil de playa durante el muestreo, no se presentó un patrón de zonación definido por ningún grupo o especie como ocurre en otras latitudes (Dahl 1952-53). Esto podría estar asociado a su propia residencia y arraigo. Posiblemente la falta de ese patrón sea causa del tiempo de residencia de la arena en muchos de los sitios donde la dinámica de erosión es intensa (Shih \& Komar 1994, Guido-Aldana et al. 2009) y donde el impacto físico por el desove de las tortugas (Xcacel), así como por el relleno que hacen algunos hoteles para mantener una playa, impiden que la fauna tenga el tiempo suficiente para evolucionar hacia una comunidad más madura y más estable. Por otra parte, la combinación del efecto de las mareas y de los vientos predominantes pueden producir presiones advectivas horizontales en las masas de agua circundantes. Esto puede repercutir de manera importante en el gradiente de humedad de la playa, apoyando la distribución a todo lo largo de la playa de algunos grupos (como los foraminíferos), que tienen una estructura física apropiada (McLachlan \& Hesp 1984, Dexter 1992).

La riqueza de especies, de los moluscos y foraminíferos fue mayor en la Riviera Maya, donde las playas son más extensas, protegidas y con alta uniformidad en su granulometría,como sucede en la mayoría de las playas de Sudamérica y en algunas del Pacífico Mexicano (Dexter 1976, Jaramillo 1994). En otras regiones existe cierto patrón en la riqueza, composición y distribución de la abundancia de la psammofauna (Brazeiro 1999), pero en este caso ese patrón fue muy dificil de observar.
La diversidad de los principales grupos (moluscos y foraminíferos), permitió distinguir entre sitios que pertenecen a la Riviera Maya y sitios que pertenecen a la Gran Costa Maya. Esta separación se relaciona con las diferentes características de esas zonas; por una parte en la Riviera Maya, se tienen playas arenosas más amplias con un tamaño de grano que va de arenas medias hasta arenas finas, por la otra en la Gran Costa Maya se tienen playas estrechas con un tamaño de grano que va desde arenas medias a arenas gruesas con escasas inclusiones de arena fina. Ambas zonas tienen una dinámica costera diferente, el oleaje es más atenuado en la Riviera Maya, donde se presenta una laguna arrecifal más amplia y donde se tiene mayor influencia turística, pero que quizá ese uso origine una perturbación media que ocasione un aumento significativo en la diversidad de algunos grupos como los moluscos (Dayton 1971, Curtis et al. 2007). Estas diferencias espaciales pueden ser el resultado de un patrón dinámico de seriación como el propuesto por Clarke et al. (1984), donde no existe una zonación estática ni definida, que refleje las diferentes capacidades de las especies existentes en los niveles, para responder a los factores ambientales prevalecientes, como ocurre en otras playas (Riascos 2002). La ausencia de algunos grupos puede estar influida por el tamaño de grano de las playas, que es fina en la Riviera Maya y más gruesa (producto de restos de conchas y corales) en la Gran Costa Maya, esto hace que algunos grupos como los moluscos y foraminíferos estén ausentes en zonas con sedimento medio-grueso, siendo frecuentes en las zonas con sedimento medio-fino. Por el contrario, los anélidos son abundantes y frecuentes en zonas de sedimento medio-grueso y ausentes o poco frecuentes en zonas con sedimento medio-fino. En esta distribución la distancia entre cada nivel de muestreo, la granulometría del sedimento, la energía del oleaje, pero sobre todo, las prácticas de relleno de playas, pudieran ser consideradas como elementos de pronóstico en la distribución de la fauna.

El ensamblaje de los sitios también permitió detectar fuertes diferencias, separando la Riviera Maya de la Gran Costa Maya, aún cuando ambas áreas están constituidas por una placa cársica y por consecuencia tienen una geología similar (CarranzaEdwards \& Nolasco-Montero 1988). Estas diferencias pueden estar determinadas por la fuerza del oleaje y por la posición de la línea de costa, ya que en la Riviera Maya hay un mayor número de playas arenosas y en la Costa Maya un mayor número de playas rocosas; sin embargo, probablemente por la cercanía, no se detectó ningún papel de la latitud en la estructuración de la psammofauna en toda el área de muestreo como lo reporta Dexter (1992). 
Los resultados confirman la gran sensibilidad de los organismos bentónicos a las condiciones ambientales prevalecientes en las playas ya que existe una gran relación entre la macrofauna bentónica, el sedimento y las características hidrodinámicas que se evidencia en la variabilidad espacial encontrada en todos los análisis entre la parte norte y sur de la Riviera Maya, en lo que pudiese también, influir el uso que se tiene de esos sitios (Beukema 1976, Möller et al. 1985)

Por otra parte, el desarrollo óptimo de los crustáceos y anélidos es propicio en niveles bajos de energía del oleaje y una dominancia de sedimentos medios-gruesos (CarranzaEdwards 2001). Por otra parte, otros grupos como los moluscos y los foraminíferos tienen un desarrollo óptimo en condiciones niveles de alta energía de niveles más cercanos al mar y con más flujos de las aguas que favorecen el incremento de la suspensión de los nutrientes, como se ha reportado en otras playas (Contreras et al. 2000, Jaramillo et al. 2001, Siemens et al. 2001).

Una formación natural que puede servir como límite entre ambas zonas son las bahías de la Ascensión y del Espíritu Santo, que son áreas muy someras protegidas del oleaje por la barrera de arrecifes coralinos, y tienen ecosistemas muy desarrollados de manglares y pantanos, que posiblemente sirvan como una barrera que disipa la corriente que viene del sur del Mar del Caimán y como una trampa de sedimentos lo que contribuye a una mayor amplitud en las playas arenosas de la Riviera Maya. La Gran Costa Maya en la parte sur al no tener una estructura semejante, está más expuesta con un mayor número de playas rocosas donde es escaso el sedimento fino, la pendiente de esas playas es en promedio de $15^{\circ}$ de inclinación y su amplitud promedio de $12 \mathrm{~m}$ (Cupul \& Téllez 1997).

En resumen, existen condiciones ambientales muy particulares tanto en la Riviera Maya como en la Gran Costa Maya y aunque algunas de ellas se asemejan, otras son diferentes. En la primera: 1) No existe una red fluvial con excepción de cenotes y ojos de agua, que aporte sedimentos a la plataforma. 2) Dominio de los factores que influyen en la sedimentación (corriente y oleaje). 3) Presencia de una baja llanura litoral, compuesta por ciénagas, lagunas costeras y una rica población de mangles, fuente importante de materia orgánica. 4) Un prevaleciente intemperismo químico y biológico. 5). Un predominio de la porción arenosa en todas las localidades, no obstante, la composición por textura es heterogénea, ya que está representada por partículas finas y medianas pero todas ellas de origen carbonatado y restos de organismos marinos. La Gran Costa Maya (zona Sur), presenta las siguientes características: 1). No existe una red fluvial con excepción del río Hondo; sin embargo, hay numerosos ojos de agua cercanos a las playas. 2). Dominio de los factores que influyen en la sedimentación (corrientes y oleaje). 3) Presencia de una baja llanura litoral, (ciénagas, lagunas costeras y mangles), fuente importante de materia orgánica. 4) Un prevaleciente intemperismo químico y biológico. 5). Presencia de zonas rocosas con alternancia de pequeñas playas arenosas, generalmente de restos biogénicos de corales y conchas. Esta mezcla de factores, hacen que la psammofauna de Quintana Roo tenga rasgos únicos que deben ser valorados e investigados a mayor detalle y a través del tiempo.

\section{LiTERATURA CITADA}

Abbott RT. 1996. Seashells of North America: A guide to field identification. A Golden Field Guide from St. Martin's Press, 280 pp. Golden Books Publishing Company, New York.

Batllori SE. 2012. Gestión para la rehabilitación de playas en la franja costera del estado de Yucatán, 21 pp. SEDUMA, Mérida. <http://www.seduma.yucatan.gob.mx/eventosmemorias/documentos_foro_erosion_costera/ Eduardo_Batllori_Seduma.pdf>

Beukema JJ. 1976. Biomass and species richness of the macrobenthic animals living on the tidal flats of the Dutch Wadden Sea. Netherland Journal of Sea Research 10: 236261.

Brazeiro A. 1999. Community patterns is sandy beaches of Chile: richness, composition, distribution and abundance of species. Revista Chilena de Historia Natural 72: 93-105.

Cano AG. 2010. Manual de prácticas de la materia de Edafología, 64 pp. Gobierno del Estado de Chiapas, Tuxtla Gutiérrez, Chiapas.

Carranza-Edwards A. 2001. Grain size and sorting in modern beach sands. Journal of Coastal Research 17(1): 38-52.

Carranza-Edwards A \& E Nolazco-Montero. 1988. Estudio sedimentológico regional de playas de Yucatán y Quintana Roo, México. Anales del Instituto de Ciencias del Mar y Limnología, UNAM, México 15(2): 49-66.

Clarke KR \& RM Warwick. 1994. Changes in marine communities: An approach to statistical analysis and interpretation, 144 pp. Natural Environment Research Council, Plymouth.

Contreras H, E Jaramillo \& P Quijon. 2000. Natural history of Emerita analoga (Stimpson) (Anomura, Hippidae) in a sandy beach of northern Chile. Revista Chilena de Historia Natural 73: 705-718.

Cupul M \& D Téllez. 1997. Variaciones espaciotemporales de la fauna macrobentónica de una playa arenosa y su relación con los cambios del perfil de playa y el tamaño de grano de los sedimentos, en playa el Pelícano, Baja California. Ciencias Marinas 23(4): 419-434.

Curtis H, NS Barnes, A Schnek \& A Massarini. 2007. Biología, 1160 pp. Editorial Médica Panamericana, México. 
Cutler EB. 1973. Sipuncula of the western North Atlantic. Bulletin of American Museum Natural History 152(3): 107204.

Dahl E. 1952-53. Some aspects of the ecology and zonation of the fauna on sandy beaches. Oikos 4(1): 1-27.

Dayton PK. 1971. Competition, disturbance and community organization: the provision and subsequent utilization of space in a rocky intertidal community. Ecological Monographs 41: 351-389.

De Huelbes A, R Guerra, E Nápoles \& ME Chávez. 1998. Foraminíferos bentónicos recientes de la plataforma insular del Archipiélago Sabana- Camagüey. Una aproximación a los factores ambientales que controlan su distribución. Memorias del III Congreso de Geología y Minería. Tomo 1: 315-318. Editorial CNDIG, La Habana.

Dexter DM. 1976. The sandy-beach fauna of Mexico. Southwestern Naturalis 20(4): 479-485.

Dexter DM. 1992. Sandy beach community structure: the role of exposure and latitude. Journal of Biogeography 19: 59-66.

Ezcurra E \& M Equihua. 1984. La teoría de la información aplicada a la clasificación de datos biológicos. En: Ezcurra E, M Equihua, GB Kohlmann \& S Sánchez (eds). Métodos cuantitativos en la biogeografía, pp. 9-40. Instituto de Ecología A.C., México.

Fauchald K. 1977. The polychaete worm's definitions and key to the orders, families and genera. Natural History Museum of Los Angeles Country, Los Angeles Science Series 28: 1188.

Felder DL. 1973. An annotated key to crabs and lobsters (Decapoda, Reptantia) from coastal waters of the Northwestern Gulf of México, 103 pp. Publication of the Center of Wetlands Resources, Baton Rouge.

Gibson R. 1972. Nemerteans, 224 pp. Hutchinson University Library, London.

Greiner GOG. 1970. Environmental factors causing distributions of recent foraminifera. Thesis Ph. D, Case Western Reserve University, Cleveland, $194 \mathrm{pp}$.

Guido-Aldana P, A Ramírez-Camperos, L Godínez-Orta, S Cruz-León \& A Juárez-León. 2009. Estudio de la erosión costera en Cancún y la Riviera Maya, México. Avances en Recursos Hidráulicos 20: 41-56.

Jaramillo E. 1994. Patterns of species richness in sandy beaches of South America. South African Journal of Zoology 29: 227234.

Jaramillo E, H Contreras, C Duarte \& P Quijon. 2001. Relationships between community structure of the intertidal macroinfauna and sandy beach characteristic along the Chilean coast. Marine Ecology 22(4): 323-342.

Loeblich A Jr \& H Tappan. 1988. Foraminiferal genera and their classification, 1728 pp. Springer Science, New York.

Magurran AE. 1988. Ecological diversity and its measurement, 179 pp. Princeton University Press, Princeton.
Martín JA \& A Rodríguez-Perea. 1996. Participación vegetal en la construcción de los sistemas dunares litorales de Mallorca. En: Grandal d'Angrade A (ed). IV Reunión de geomorfología, pp. 785-789. Sociedad Española de Geomorfología, O. Castro.

McLachlan A \& P Hesp. 1984. Faunal response to morphology and water circulation of a sandy beach with cusps. Marine Ecology Progress Series 19: 133-144.

Möller P, L Pihl \& R Rosenberg. 1985. Benthic faunal energy flow and biological interaction in some shallow marine soft bottom habitats. Marine Ecology Progress Series 27: 109121.

Orlóci L. 1978. Multivariate analysis in vegetation research, 97 pp. Dr. W.J. Junk Publishers, The Hague.

Pielou EC. 1984. The interpretation of ecological data: A premier on classification and ordination, $235 \mathrm{pp}$. Wiley Interscience, New York.

Powers LW. 1977. A catalogue and bibliography to the crabs (Brachyura) of the Gulf of México, 190 pp. Port Aransas Marine Laboratory, University of Texas Marine Science Institute, Port Aransas.

Riascos JM. 2002. Cambios en el macrobentos de la playa arenosa durante 'El Niño' 1997-98 en la bahía de Málaga, Pacifico colombiano. Ciencias Marinas 28(1): 13-25.

Roig FX. 2003. Identificación de variables útiles para la clasificación y gestión de calas y playas: el caso de la isla de Menorca (I. Balears). Boletín de la AGE 35: 175-190.

Schmitt T. 1994. Degradació de la vegetació psamófila litoral de Mallorca. Boletín de la Sociedad de Historia Natural de Baleares 37: 151-174.

Shih S \& P Komar. 1994. Sediments, beach morphology and sea cliff erosion within an Oregon coast littoral cell. Journal of Coastal Research 10(1): 144-157.

Siemens R, SM Mudge \& JM Cancino. 2001. The effect of physical and chemical parameters on the macroinfaunal community structure of San Vicente Bay, Chile. Revista Chilena de Historia Natural 74(2): 429-444

Stephen AC \& SJ Edmonds. 1977. The phyla Sipuncula and Echiura, 528 pp. British Museum (Natural History), London.

Torruco D \& A González-Solís. 2012. Evaluación de las playas del litoral de Quintana Roo: Extensión y biodiversidad. Revista Innovación para la Vinculación FOMIX-Quintana Roo 2(4): 51-61.

Torruco D, A González-Solís \& AD Torruco-González. 2013. Las playas de Quintana Roo: sus riesgos y vulnerabilidad. El Periplo Sustentable 24: 155-172.

Warmke GL \& RT Abbot. 1962. Caribbean seashells a guide to the marine mollusks of Puerto Rico and other West Indian Island, Bermuda and the Lower Florida Keys, 348 pp. Dover Publications, New York

Williams AB. 1984. Shrimps, lobsters and crabs of the Atlantic coast of the Eastern United States, Maine to Florida, 552 pp. Smithsonian Institute Press, Washington. 
Anexo 1. Presencia de especies en las playas de Quintana Roo, México / Species presence on the Quintana Roo, Mexico beaches

\begin{tabular}{lllllllllll} 
Playas arenosas & 1 & 2 & 3 & 4 & 5 & 6 & 7 & 8 & 9 & 10 \\
\hline
\end{tabular}

Mollusca

Anomia simplex D’Orbigny, 1853

Parvanachis obesa (C.B. Adams, 1813)

Divalinga quadrisulcata (D’Obrigny, 1846)

Pristiterebra tuberculosa (Hinds, 1844)

Epitonium apiculatum (Dall, 1889)

Rissoella caribaea Rohder, 1943

Turbo castanea Gmelin, 1791

Lucapinella limatula (Reeve, 1850)

Eulithidium affine (C.B. Adams, 1850)

Melanphus coffea (Linnaeus, 1758)

Bentonella tenella (Jeffreys, 1869)

Sanguinolaria cruenta (Lightfood, 1786)

Vermetidae incertae sedis irregularis D’Orbigny, 1841

Assiminea succinea (Pfeiffer, 1840)

Volvarina avena (Kiener, 1834)

Eulithidium bellum (M. Smith, 1937)

Tellina listeri Röding, 1798

Smaragdia viridis (Linnaeus, 1758)

Crustacea

Excirolana mayana (Ives, 1891)

Eurydice convexa Richardson, 1900

Gammarus annulatus Smith, 1873

Talitrus saltator (Montagu, 1808)

Annelida

Arenicola cristata Stimpson, 1856

Scoloplos latum (Chamberlin, 1919)

Pseudonereis variegata (Grube, 1857)

Scolelepis squamata (Müller, 1806)

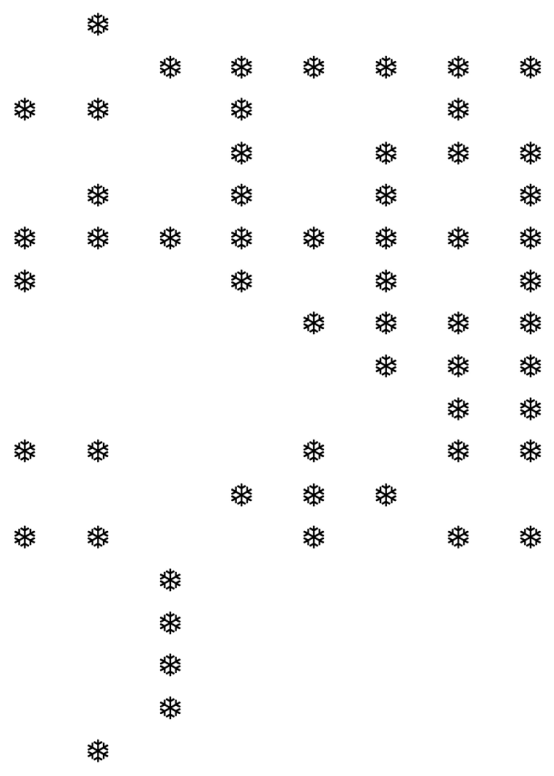

Sipuncula

Antillesoma antillarum (Grube, 1858)

Nemertea

Prostoma sp.

Foraminifera

Archaias agulatus (Fichtel \& Moll, 1798)

Cyclorbiculina compressa (D’Orbigny, 1839)

Bigenerina angulata Cushman, 1927

Homotrema rubra (Lamarck, 1816)

Insecta

Formicidae

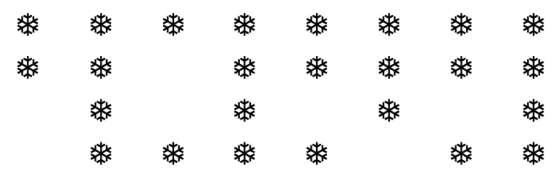

